\title{
Stimulus duration and partial report performance
}

\author{
JAMES M. YEOMANS and DAVID E. IRWIN \\ Cornell University, Ithaca, New York
}

\begin{abstract}
This paper investigates the effect of stimulus duration on partial report performance. Two experiments showed that variations in stimulus duration from 50 to $500 \mathrm{msec}$ had little or no effect on partial report accuracy, decay, or error patterns. These results contrast with previous research demonstrating that increasing stimulus duration decreases visible persistence duration. Our findings thus provide support for the argument (Coltheart, 1980) that informational persistence (knowledge about the visual properties of a stimulus) is a separate phenomenon from visible persistence (the phenomenal persistence of a stimulus), and suggest that the traditional view of iconic memory, which conflates these two forms of persistence, is incorrect. Furthermore, our results indicate that informational persistence is not merely a function of unnaturally brief stimulus exposures, but exists even after exposure durations as long as $500 \mathrm{msec}$. Possible mechanisms of informational persistence, as well as its potential role in everyday perception, are discussed.
\end{abstract}

It has been known for centuries that visual sensation persists after stimulus offset; the writings of Aristotle (384-322 B.C.) reportedly contain the first known reference to this phenomenon (Allen, 1926). The fairly recent rekindling of interest in visual persistence can, perhaps, be traced to the work of Sperling (1960). Sperling briefly presented subjects an array of letters and, following their offset, cued report of one row of the array. Sperling found that subjects' recall performance for the cued row was very high if the cue was presented within about $100 \mathrm{msec}$ or so of stimulus offset. Furthermore, recall accuracy decreased as the time between stimulus offset and presentation of the recall cue increased. These results stood in contrast with what happened when subjects were asked to report the entire array of letters. In this case, recall performance was limited to only a few items from the array. Taken together, these results suggested that immediately following stimulus offset there was more information available about the array than could be normally reported, but this information disappeared quickly with the passage of time. This method of sampling a subset of the total information in an array has been called the partial-report technique, and the superior recall performance under these conditions, the partial-report superiority effect.

Preparation of this manuscript was supported by an All University Research Initiation Grant from Michigan State University to the second author. Experiment 1 was part of a Senior Honors Thesis submitted by the first author to Cornell University. We thank C. W. Eriksen and Vince Di Lollo for their comments on an earlier version of the manuscript, Jennifer Freyd and Thomas Gilovich for their comments on the research, Sun Jun-Shi for programming assistance, Brian Engler and Michael Tarr for help with the data analysis, and Frank Keil and Ulric Neisser for intellectual stimulation. Both authors are now affiliated with Michigan State University. Requests for reprints should be sent to David E. Irwin, Department of Psychology, Michigan State University, East Lansing, MI 48824.
Although Sperling's study demonstrated the persistence of information from a letter array, the evidence that this information was visual was somewhat indirect. One source of evidence was subject's introspective reports that they were "reading" a slowly fading stimulus trace; a somewhat more compelling piece of evidence was that the visual characteristics of the exposure fields presented before and after the stimulus array had a sizable effect on the rate of decay of information from the array. Specifically, with a dark pre- and postexposure field, partialreport performance was better than whole-report performance for cue delays longer than $2 \mathrm{sec}$; this superiority was limited to about $0.5 \mathrm{sec}$ when pre- and postexposure fields were about the same intensity as the stimulus array.

Several other studies have provided additional evidence that the information sampled by the partial-report technique is visual in nature. These studies manipulated the physical characteristics of the items in the stimulus array and the nature of the partial report cue. In general, partialreport cues that select items on the basis of some visuospatial characteristic yield a partial-report superiority, whereas those that require some classification of the stimuli do not. For example, location (e.g., Sperling, 1960), color (Clark, 1969), brightness (von Wright, 1968), and shape (Turvey \& Kravetz, 1970) have all been found to act as effective partial-report cues. However, if the partial-report cue is to report just the letters from an array of letters and digits (Sperling, 1960; von Wright, 1968), or to report only the letters that end in "ee" (Coltheart, Lea, \& Thompson, 1974), then no partial-report superiority is found. These results suggest that the information that persists from a stimulus array, and that the partial report technique measures, has only visual, and not semantic or phonological, characteristics.

Several, more "direct," methods of investigating visual persistence have also been developed. Sperling (1967), 
for example, introduced a technique for measuring the phenomenal duration of a stimulus by adjusting the occurrence of a probe so that its onset and offset appear synchronous with stimulus onset and offset. Estimates of persistence duration obtained with this method approximate those obtained from partial-report experiments (Haber \& Standing, 1970). Another direct method involves presenting a stimulus discontinuously in time and varying the interval between presentations; the stimulus appears continuous as long as its persistence outlasts the blank period between presentations. Haber and Standing (1969) and Meyer, Lawson, and Cohen (1975) found persistence duration in this task to be quite similar to partial-report estimates. Eriksen and Collins $(1967,1968)$ introduced a variant of this technique in which two random-dot patterns were presented sequentially, separated by an interstimulus interval. When superimposed, these patterns formed a nonsense syllable. Eriksen and Collins (1967) found that subjects could temporally integrate the two dot patterns to perceive the nonsense syllable over intervals as long as $100 \mathrm{msec}$, yielding an estimate of persistence duration approximating that obtained from partial report. The results of these direct measurement studies, along with those from partial-report experiments, led to the consensual notion (e.g., Coltheart et al., 1974; Dick, 1974; von Wright, 1972) that visual information persists after stimulus offset in a precategorical, high-capacity, quickly decaying memory store, which Neisser (1967) called "iconic memory."

Recently, this traditional view of iconic memory has come under attack. Several sources of evidence suggest that the stimulus persistence measured by the partial-report technique is identifiably different from that measured by the more direct techniques. In particular, it appears that the partial-report technique might access more than just raw stimulus persistence. Two pieces of evidence support this conjecture. One is that most errors in partialreport tasks are mislocation errors, rather than itemintrusion errors (Townsend, 1973). This fact suggests that items in the array are identified and remembered quite well, but their locations are forgotten. In addition, familiarity with the stimulus array has been found to reduce the number of item, but not location, errors (Mewhort, Campbell, Marchetti, \& Campbell, 1981). These two findings suggest that the partial-report technique measures not just raw sensory persistence, but also pattern recognized information. Further evidence that information accessed by partial report is different from that measured by direct persistence techniques is provided by the finding that performance on these two kinds of tasks is differentially affected by stimulus factors such as stimulus intensity. For example, tasks that require visible stimulus persistence regularly show an inverse relationship between stimulus intensity and persistence duration (e.g., Bowen, Pola, \& Matin, 1974; Efron, 1970a; Haber \& Standing, 1969), but partial-report experiments do not (e.g., Adelson \& Jonides, 1980; Eriksen \& Rohrbaugh, 1970; Scharf \& Lefton, 1970). These findings have led several investigators (e.g., Coltheart, 1980; Di Lollo,
1980; Mewhort et al., 1981) to challenge the widely held notion that all information about a stimulus persists in a single, precategorical, high-capacity, quickly decaying iconic memory. Coltheart (1980), for example, distinguishes among three types of persistence that follow stimulas offset: neural persistence, due to residual activity in the visual pathway; visible persistence, or the phenomenal impression that the stimulus is still visibly present; and informational persistence, or knowledge about the visual properties of the stimulus. Under the traditional view of iconic memory, these three forms of persistence have been equated with one another; Coltheart's contention is that visible persistence must be different from informational persistence, since visible persistence is affected by stimulus intensity, while informational persistence (which is all that partial report measures) is not.

One purpose of the experiments reported in this paper was to further demonstrate that visible persistence and informational persistence are separate phenomena. This was accomplished by manipulating stimulus duration. The effects of stimulus duration on visible persistence are well known: persistence duration decreases as stimulus duration increases (Bowen et al., 1974; Cohene, 1975; Di Lollo, 1977, 1980; Efron, 1970a, 1970b, 1970c; Haber \& Standing, 1970). The effect of stimulus duration on partial report is less well known. Only two studies have examined it. Sperling (1960) found no difference in partial report between exposure durations of 15 and $50 \mathrm{msec}$. Di Lollo (1978) found no difference between 100 - and 200-msec-exposure durations for immediate report, but he did not investigate potential differences in decay rate. So the effect of stimulus duration on partial report for a range of exposure durations and cue delays is essentially unknown. Demonstrating that stimulus duration has differential effects on informational and visible persistence would provide support for Coltheart's argument, and would indicate that theories of early visual memory need to be revised to account for these different levels of coding.

The effect of stimulus duration on partial report is significant for another reason, as well. Iconic memory has been studied almost exclusively for extremely brief (usually 50-msec) exposure durations that rarely occur in the natural environment. This has led some researchers to question whether visual persistence plays any role in normal perception. Haber (1983), for example, states: "Brief discrete flashes have a visual persistence. But normal perception is not made up of brief discrete flashes, singly or in combination. Persistence, and therefore icons, are irrelevant' (p. 3). Demonstrating that informational persistence exists even after long exposure durations that approximate those of typical eye fixations would vitiate this criticism and would invite theoretical discussion of its role in normal perception.

\section{EXPERIMENT 1}

\section{Method}

Subjects. Six Cornell University undergraduates participated in 
this experiment. All subjects had normal or corrected-to-normal vision. They were all paid $\$ 3$ for each of two $1-h$ sessions.

Apparatus. A two-field Harvatd tachistoscope (Model T-2B-1) was used to present the stimuli and partial-report cues. The stimuli consisted of $1003 \times 3$ letter arrays printed on $4 \times 6$ in. white matte cards. A Hewlett-Packard graphics plotter was used to make the stimuli. The letters were constructed from the duplex type font. All letters were used except for vowels and the letter y. Each letter array subtended $3.5^{\circ}$ of visual angle vertically and $3.4^{\circ}$ horizontally when presented. Letters were $0.6^{\circ}$ high and $0.4^{\circ}$ wide. Horizontal spacing between letters was $1^{\circ}$, and the vertical spacing was $0.9^{\circ}$. Three partial-report cue cards were also constructed. Each card contained an arrow pointer that aligned with one of the three rows. The arrow subtended $0.6^{\circ}$ of visual angle in both the horizontal and vertical dimensions, and appeared $1.2^{\circ}$ away from the left side of the letter array when presented. The stimuli and partial-report cues were presented at a luminance of $16 \mathrm{fL}$. The experimental area was kept dark throughout the experiment, except for a small desk lamp which allowed the experimenter to enter the cards into the tachistoscope and record the subjects' responses.

Procedure. On each experimental trial, a $3 \times 3$ letter array was presented for some duration, then some period of time later the arrow pointer appeared to cue one of the rows for report. The subjects were instructed to report the three letters in the indicated row in their proper spatial order, guessing if unsure. The experimenter recorded this response for each trial. The subjects initiated each trial by pressing a triggering lever after the experimenter had indicated that the cards were in place.

Two exposure durations (50 and $200 \mathrm{msec}$ ) and five cue delays $(0,50,150,300$, and $500 \mathrm{msec})$ were employed in a completely crossed design. The pre- and postexposure fields consisted of a dark field. The partial-report cue was presented for $50 \mathrm{msec}$.

The experiment consisted of 10 blocks of 30 trials each. Each block contained only a single duration-interval pairing. The first five trials of each block were considered as practice, and discarded. Five blocks were run during each of two $1-h$ sessions. The order in which the 10 duration-interval pairings were presented was randomize for each subject.

\section{Results and Discussion}

On each trial the subjects made three responses, corresponding to the three letters they thought had been presented in the cued row. Each of these responses was scored as either a correct report (if the correct letter was reported in the correct position), a location error (if a letter from the $3 \times 3$ stimulus display was reported, but in the incorrect position), or an intrusion error (if a letter not contained in the $3 \times 3$ stimulus display was reported). Figure 1 shows the proportions of these responses for this experiment as a function of stimulus duration and cue delay. Separate analyses of variance were performed for each of these measures.

For correct reports, only the main effect of cue delay was significant $[\mathrm{F}(4,20)=23.78, \mathrm{p}<.001]$. Correct reports declined the longer the cue was delayed, regardless of stimulus duration. The stimulus duration main effect was not significant $[F(1,5)=0.01, p>.9]$, nor was the stimulus duration $\times$ cue delay interaction $[F(4,20)=$ $1.08, \mathrm{p}>.3]$.

In the analysis of intrusion errors, only the main effect of cue delay was significant $[F(4,20)=12.65$, $\mathrm{p}<.001]$. Stimulus duration $[\mathrm{F}(1,5)=0.26, \mathrm{p}>.6]$ and the stimulus duration $\times$ cue delay interaction $[\mathrm{F}(4,20)$ $=1.27, \mathrm{p}>.3 \mathrm{]}$ were not significant. The same pattern held for location errors: cue delay $[F(4,20)=9.75$,

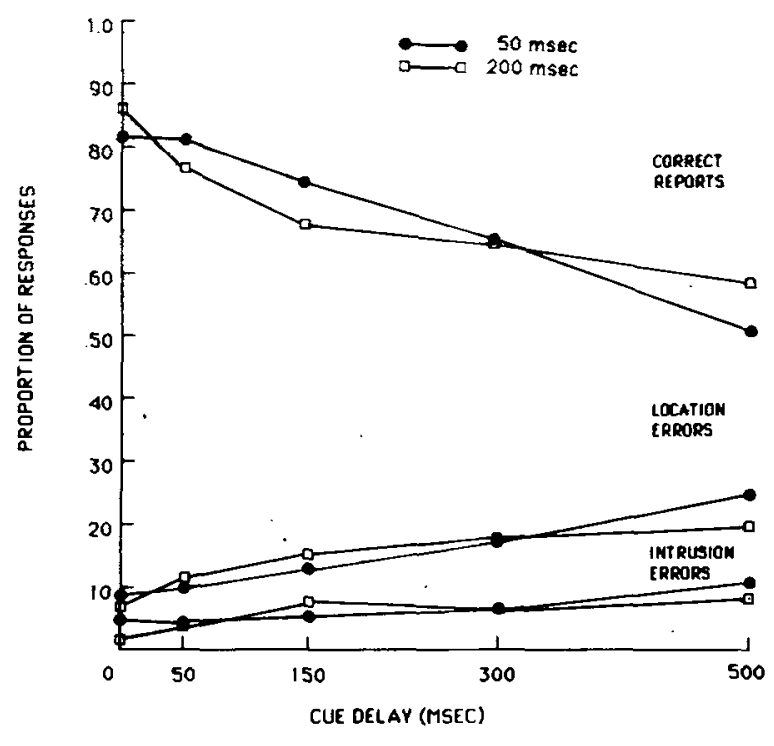

Figure 1. Correct reports, location errors, and intrusion errors as a function of cue delay and stimulus duration in Experiment 1.

$\mathrm{p}<.001]$ was significant, but stimulus duration $[\mathrm{F}(1,5)$ $<0.01, p>.9]$ and the stimulus duration $\times$ cue delay $[F(4,20)=0.26, p>.8]$ interaction were not. There were more location errors than intrusion errors at each cue delay. Both types of errors increased as cue delay increased, although intrusions did so at a slower rate.

In summary, correct reports decreased and both types of errors increased as the interval between stimulus offset and cue presentation was lengthened. Most importantly, stimulus duration had no effect on overall accuracy, decay of the partial report superiority, or on the pattern of errors. These results stand in striking contrast with results obtained from visible persistence experiments. Di Lollo (1980), for example, found that accuracy in a temporal integration task decreased dramatically as stimulus duration increased from 10 to $200 \mathrm{msec}$. Furthermore, subjects' error patterns also changed radically as stimulus duration increased. The fact that stimulus duration has such differential effects on partial report and visible persistence tasks supports the view that they measure separate processes, as Coltheart (1980) and others have argued.

Furthermore, the results of this experiment demonstrate that informational persistence exists even for exposure durations that approximate those of normal eye fixations. Informational persistence occurs not only for very brief exposures, but for fixation-length exposures as well. Since much of our visual input about the world is garnered via successive, discrete eye fixations, informational persistence might well be relevant to the study of natural perception.

\section{EXPERIMENT 2}

Experiment 2 is a replication and extension of Experiment 1 . Stimulus durations of 50,200 , and $500 \mathrm{msec}$ were used. If this range of exposure durations caused no 
differential effects on partial-report performance, the arguments that visible and informational persistence are separate and that informational persistence exists for fixation-length stimulus durations would be greatly strengthened.

\section{Method}

Subjects. Six subjects participated in this experiment. All had normal or corrected-to-normal vision. None had participated in the first experiment.

Apparatus. Stimuli were presented on a Tektronix $5103 \mathrm{~N}$ oscilloscope equipped with P31 phosphor. A Digital Equipment Corporation PDP-1 1/24 computer controlled stimulus presentation via digital-to-analog converters. As in Experiment 1, the stimuli consisted of $3 \times 3$ letter arrays constructed from the set of all consonants excluding $y$. The partial-report cue was a square presented to the side of one row. All visual angles in this experiment were identical to those used in Experiment 1. The experimental chamber was illuminated in order to prevent subjects from detecting phosphor decay.

Procedure. On each trial, subjects were presented a $3 \times 3$ letter array for 50,200 , or $500 \mathrm{msec}$. After the array was extinguished, an interval of $0,50,150,300$, or $500 \mathrm{msec}$ elapsed before the partial report cue was presented. The subjects then typed their responses into the computer. The subjects initiated each trial by pressing the return key on the keyboard.

The experiment consisted of five blocks of 45 trials each. The three exposure durations and five cue delays were completely crossed, and then their order of presentation was randomized across trials. The subjects completed a practice block of 45 trials, then the five experimental blocks, in a single session lasting about $45 \mathrm{~min}$.

\section{Results and Discussion}

The subjects' responses were scored as in Experiment 1 . Figure 2 shows the results (correct reports, location errors, and intrusion errors) for this experiment. Separate analyses of variance were performed for each of these measures.

In the analysis of correct reports, only the main effect of cue delay was significant $[F(4,20)=10.23, p<.001]$. Correct reports declined as cue delay increased, for all stimulus durations. The duration main effect was not significant $[F(2,10)=1.71, p>.2]$, although correct reports did increase slightly as stimulus duration increased $(57.7 \%$ vs. $61.3 \%$ vs. $63.0 \%$ for $50-, 200$-, and 500 -msec exposure durations). The duration $\times$ cue delay interaction was also not significant $[F(8,40)=1.31, p>.2]$, indicating that there were no reliably systematic differences in the partial-report decay functions for the various exposure durations.

In the analysis of intrusion errors, the cue delay main effect was significant $[F(4,20)=4.23, p<.025]$. There were more intrusion errors at longer than at shorter delays. The stimulus duration main effect was also significant in this experiment $[\mathrm{F}(2,10)=5.61, \mathrm{p}<.025]$. There were significantly more intrusion errors at the 50 msec stimulus duration $(20.8 \%)$ than at $500 \mathrm{msec}$ (16.4\%). The difference between 50-msec $(20.8 \%)$ and $200 \mathrm{msec}(16.9 \%)$ approached significance (Scheffe confidence interval half-width $=4.2 \%$ ). The interaction between cue delay and stimulus duration was not significant $[F(8,40)=1.02, p>.4]$. In the analysis of location

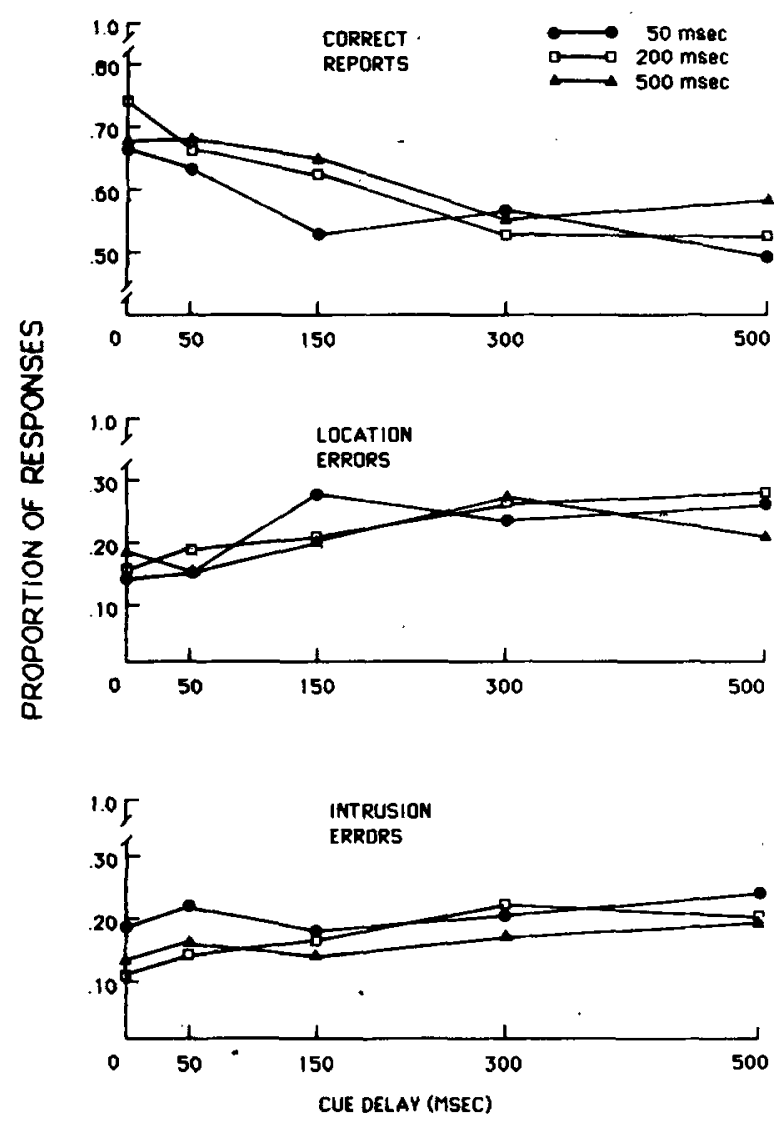

Figure 2. Correct reports, location errors, and intrusion errors as a function of cue delay and stimulus duration in Experiment 2.

errors, only the main effect of cue delay was significant $[F(4,20)=8.12, p<.001]$. Location errors increased as cue delay increased. The stimulus duration main effect was not significant $[F(2,10)=0.13, p>.8]$, nor was the stimulus duration $\times$ cue delay interaction $[F(8,40)$ $=1.40, \mathrm{p}>.2]$.

In summary, as in Experiment 1, stimulus duration had little effect on overall accuracy or on decay of the partialreport superiority. There was, in this experiment, a suggestion of improved accuracy with increasing stimulus duration, but the improvement was small and nonsignificant. Stimulus duration did affect intrusion errors in this experiment, unlike in Experiment 1, such that fewer intrusion errors were made as stimulus duration increased. Since stimulus duration varied from trial to trial in this experiment, it may be that the 50 -msec exposures were relatively less perceptible than the 200 - and $500-\mathrm{msec}$ exposures, so that subjects were more often unsure what letters had been presented on 50-msec exposure trials. The fact that stimulus duration affected only intrusion, and not location, errors is consistent with this explanation. In Experiment 1, stimulus duration was kept constant for an entire block of trials, so subjects might have become accustomed to whatever exposure duration was used. Regardless of whether increasing stimulus duration has no or only a slightly positive effect on partial report per- 
formance, its influence here is still vastly different from its influence on visible persistence, where an increase in stimulus duration causes a decrease in persistence duration. It is again also clear from this experiment that informational persistence is not merely an artifact of extremely brief exposure durations; rapidly decaying information about the contents of a visual display persists even after exposures as long as $\mathbf{5 0 0}$ msec.

\section{GENERAL DISCUSSION}

The experiments reported in this paper provide support for the contention that informational persistence (i.e., knowledge about the visual properties of a justextinguished stimulus) is a separate phenomenon from visible persistence (the phenomenal visibility of a justextinguished stimulus). Stimulus duration and stimulus intensity are known to have large inverse effects on visible persistence duration, but these same variables appear to have little or no effect on informational persistence as measured by a partial-report task. The traditional view of iconic memory, which equates visible and informational persistence, needs revision.

One possibility is a two-stage model in which a brief period of visible persistence is followed by a longer period of informational persistence. The period of visible persistence would begin with stimulus onset, and its duration would depend upon stimulus duration and stimulus intensity. Following stimulus offset, a period of informational persistence would begin. The characteristics of this second stage, as suggested by the current study, are the following: (1) a representation of the spatial arrangement of the extinguished display that is initially very accurate, but rapidly decays to asymptote about $300 \mathrm{msec}$ after stimulus offset (actually, the period of informational persistence probably lasts even some $200-300 \mathrm{msec}$ longer than this, since perception and interpretation of the partialreport cue take some time; Colegate, Hoffman, \& Eriksen, 1973; Eriksen \& Collins, 1969); (2) loss of location information about the display earlier, and at a faster rate, than identity information; and (3) no effect of display duration on persistence, as long as the display is presented long enough for identification of its contents to occur.

In the two-stage model outlined above, visible persistence is viewed as a byproduct of neural persistence in the visual pathway. The source of informational persistence is less clear. One possibility that is consistent with the three characteristics described above is that visual information persists in a nonvisible visual memory that begins at stimulus offset and lasts for 300-600 msec, independently of exposure duration. This memory might consist of a visual analog of the stimulus display. Drift of the elements in the analog representation, in conjunction with passive decay, would be sufficient to produce the pattern of location and identity errors that were found. This "visual" conception of informational persistence is consistent with research showing that the visual characteristics of pre- and postexposure fields have a large ef- fect on partial report performance (e.g., Averbach \& Coriell, 1961; Sperling, 1960). However, another possible conception of informational persistence that is also consistent with the three characteristics described above is that informational persistence arises from a nonvisual memory that contains spatial coordinates for items in a display along with abstract identity codes for those items. Both sources of information might decay rapidly in this memory, with faster decay for the spatial coordinates. Further research is required to discriminate between these two classes of models.

The second model described above is in some ways similar to one proposed by Di Lollo $(1978,1980)$ for memory of brief visual displays. In Di Lollo's model, a sensory recruiting phase which is onset-activated, retinotopically organized, and energy dependent is followed by a nonvisible interpretation phase in which display items are identified and categorized. During the interpretation phase, retinal and spatial information about elements in the display are lost. The major difference between the second model outlined above and Di Lollo's model concerns when spatial information about the display is lost; in Di Lollo's model, this process is dependent on stimulus onset, since interpretation immediately follows recruiting and may be finished before stimulus offset. In the second model outlined above, loss of spatial information does not begin until after stimulus offset. The experiments reported in the current paper found that loss of spatial information was contingent on display offset, independently of display onset. This aspect of the data is thus inconsistent with Di Lollo's formulation. It should be noted, however, that Di Lollo was referring to the loss of fine spatial information, such as that required for aligning individual dots in a dot matrix, rather than to the relatively gross location information required in the present task.

Another recently proposed model that is similar to the second model outlined above is the dual-buffer model of Campbell and Mewhort (1980; see also Mewhort et al., 1981). In this model, information from a display is first stored in a raw, precategorical form in a feature buffer; this raw information is transformed by a character identification mechanism into an abstract, postcategorical representation that is stored in a character buffer, which also preserves the relative spatial positions of items in the display. According to the dual-buffer model, identification errors occur predominantly at the feature buffer level, due to data corruption, whereas localization errors occur predominantly at the characer buffer level, due to retrieval failure. The dual-buffer model appears to account for all the results found in the present paper, since it accurately predicts that the only effect of exposure duration should be on intrusion errors (specifically, that there should be more intrusion errors at shorter, as opposed to longer, durations), whereas the major effect of cue delay should be on location errors.

The other major conclusion to be drawn from the research reported in this paper is that a partial-report superiority (and thus, informational persistence) exists even 
after exposure durations that last as long as typical eye fixations. In fact, the duration of informational persistence was independent of changes in exposure duration. Such constancy is important for informational persistence to play any role in natural perception, since fixation durations normally vary.

Visual persistence is not merely a consequence of artificially brief exposure durations-it is a natural phenomenon. It is likely that this persistence is not visible, but an overlapping visible persistence of successive fixations might actually hinder normal perception (Haber, 1983; Hochberg, 1968; Neisser, 1967). Informational persistence, on the other hand, could play a helpful role in perception by mediating the integration of information from successive fixations, especially during reading. A form of visual persistence that represented the identity of elements in a fixation, along with their relative spatial positions, could serve to bridge the interval of saccadic suppression that separates fixations. Rayner, McConkie, and Zola (1980) have provided evidence for just such a memory in reading. In their experiments, a word was presented in the visual periphery and subjects were instructed to move their eyes to it. During the eye movement, this word was replaced by a word which subjects read. Rayner et al. found that the initially presented word facilitated naming of the second word when the two words shared the same beginning letters, regardless of their case. Rayner et al. concluded that some nonvisible letter-identity code from the first word was stored and integrated with the word presented after the saccade. In other terms, informational persistence from the first word facilitated recognition of the target word. However, recent work by McConkie, Zola, Blanchard, and Wolverton (1982) suggests that integration of peripheral and foveal letter information does not occur when subjects read meaningful, connected text, since changes in letter identities across fixations go unnoticed under these conditions. Thus, the role of informational persistence in reading is an open question.

In conclusion, the research reported above has provided support for the argument that visible and informational persistence are separate phenomena, and that informational persistence exists under ordinary viewing conditions. Furthermore, it is possible that informational persistence may play an important role in everyday perception in the integration of information from successive eye fixations.

\section{REFERENCES}

AdELSON, E. H., JoNIDEs, J. (1980). The psychophysics of iconic storage. Journal of Experimental Psychology: Human Perception and Performance, 6, 486-493.

AlleN, F. (1926). The persistence of vision. American Journal of Physiological Optics, 7, 439-457.

Averbach, E., \& Coriell, E. (1961). Short-term memory in vision. Bell System Technical Journal, 40, 309-328.

Bowen, R. W., Pola, J., MAtin, L. (1974). Visual persistence: Effects of flash luminance, duration, and energy. Vision Research, 14, 295-303.
Campbell, A. J., \& Mewhort, D. J. K. (1980). On familiarity effects in visual information processing. Canadian Journal of Psychology, 34, 134-154.

CLARK, S. E. (1969). Retrieval of color information from preperception memory. Journal of Experimental Psychology, 82, 263-266.

Cohene, L. S. (1975). Iconic memory of dot patterns: Preliminary report. Perceptual and Motor Skills, 41, 167-170.

Colegate, R. L., Hoffman, J. E., \& Eriksen, C. W. (1973). Selective encoding from multielement visual displays. Perception \& Psychophysics, 14, 217-224.

ColthearT, M. (1980). Iconic memory and visible persistence. Perception \& Psychophysics, 27, 183-228.

ColtheArT, M., LeA, C. D., \& Thompson, K. (1974). In defence of iconic memory. Quarterly Journal of Experimental Psychology, 26, 633-641.

Dick, A. O. (1974). Iconic memory and its relation to perceptual processes and other mechanisms. Perception \& Psychophysics, 16, 575-596.

Di LoLLo, V. (1977). Temporal characteristics of iconic memory. $\mathrm{Na}$ ture, 267, 241-243.

Di LoLLo, V. (1978). On the spatio-temporal interactions of brief visual displays. In R. H. Day \& G. V. Stanley (Eds.), Studies in perception (pp. 39-55). Perth: University of Western Australia Press.

Di Lollo, V. (1980). Temporal integration in visual memory. Journal of Experimental Psychology: General, 109, 75-97.

EFron, R. (1970a). Effect of stimulus duration on perceptual onset and offset latencies. Perception \& Psychophysics, 8, 231-234.

EFron, R. (1970b). The minimum duration of a perception. Neuropsychologia, 8, 57-63.

EFroN, R. (1970c). The relationship between the duration of a stimulus and the duration of a perception. Neuropsychologia, 8, 37-55.

ERIKSEN, C. W., \& Collins, J. F. (1967). Some temporal characteristics of visual pattern perception. Joumal of Experimental Psychology, 74, 476-484.

ERIKSEN, C. W., \& Collins, J. F. (1968). Sensory traces versus the psychological moment in the temporal organization of form. Joumal of Experimental Psychology, 77, 376-382.

Eriksen, C. W., \& Collins, J. F. (1969). The temporal course of selective attention. Joumal of Experimental Psychology, 80, 254-261.

ERIKSEN, C. W., \& RoHrbaugh, J. W. (1970). Visual masking in multielement displays. Journal of Experimental Psychology, 83, 147-154.

HABER, R. N. (1983). The impending demise of the icon: A critique of the concept of iconic storage in visual information processing. Behavioral and Brain Sciences, 6, 1-54.

HABER, R. N., \& STANDING, L. (1969). Direct measures of short-term visual storage. Quarterly Journal of Experimental Psychology, 21, 43-54.

HABER, R. N., Standing, L. (1970). Direct estimates of the apparent duration of a flash. Canadian Joumal of Psychology, 24, 216-229.

Hochberg, J. (1968). In the mind's eye. In R. N. Haber (Ed.), Contemporary theory and research in visual perception (pp. 309-331). New York: Holt, Rinehart, \& Winston.

McConkie, G. W., Zola, D., Blanchard, H. E., \& Wolverton, G. S. (1982). Perceiving words during reading: Lack of facilitation from prior peripheral exposure. Perception \& Psychophysics, 32, 271-281.

Mewhort, D. J. K., Campbell, A. J., Marchetti, F. M., \& CampBELL, J. I. D. (1981). Identification, localization, and "iconic" memory: An evaluation of the bar-probe task. Memory \& Cognition, 9, 50-67.

Meyer, G. E., LAwson, R. L., \& Cohen, W. (1975). The effects of orientation-specific adaptation on the duration of short-term visual storage. Vision Research, 15, 569-572.

NeISSER, U. (1967). Cognitive psychology. New York: AppletonCentury-Crofts.

RAYNER, K., MCConkie, G. W., \& Zola, D. (1980). Integrating information across eye movements. Cognitive Psychology, 12, 206-226.

SCHARF, B., LEFTON, L. A. (1970). Backward and forward masking as a function of stimulus and task parameters. Journal of Experimental Psychology, 84, 331-338. 
SPERLING, G. (1960). The information available in brief visual presentations. Psychological Monographs, 74(11, Whole No. 498).

SPERLING, G. (1967). Successive approximation to a model for shortterm memory. Acta Psychologica, 27, 285-292.

TownsEND, V. M. (1973). Loss of spatial and identity information following a tachistoscopic exposure. Journal of Experimental Psychology, 98, 113-118.

TURVEY, M. T., \& Kravetz, S. (1970). Retrieval from iconic memory with shape as the selection criterion. Perception \& Psychophysics, 8, 171-172.
VON WRIGHT, J. M. (1968). Selection in immediate visual memory. Quarterly Journal of Experimental Psychology, 20, 62-68.

VON WRIGHT, J. M. (1972). On the problem of selection in iconic memory. Scandinavian Journal of Psychology, 13, 159-171.

(Manuscript received October 9, 1984;

Revision accepted for publication January 28, 1985.) 